\title{
Synthesis and Biological Activity of Some Schiff Bases from Phthalimides
}

\author{
Girish Kumar Sinha ${ }^{1, *}$, Akanksha Priya ${ }^{2}$, Sonal Priya ${ }^{3}$, Satyendra Narayan ${ }^{1}$ \\ ${ }^{1}$ Post Graduate Centre of Chemistry, College of Commerce, Arts and Science Patna (M.U.), India \\ ${ }^{2}$ Department of Botany, University of Delhi, India \\ ${ }^{3}$ Department of Chemistry, Oklahoma State University, USA
}

Copyright $@ 2017$ by authors, all rights reserved. Authors agree that this article remains permanently open access under the terms of the Creative Commons Attribution License 4.0 International License

\begin{abstract}
An innovative protocol to the synthesis of this material emerged on exploring the potential of the various form of $\mathrm{N}$-aminophthalimides on its reaction with a number of aromatic aldehydes. New series of biologically active substituted Schiff bases with general formula, R1N=CHR2 where R1 = 3-nitro- $\mathrm{N}$-aminophthalimide,

3-bromo-N-aminophthalimide, 4-nitro-N-aminophthalimide, 4-bromo-N-aminophthalimide, R2 =2,

6-dichlorobenzaldehyde, o-anisaldehyde and o-vanillin were synthesized by the reaction of substituted $\mathrm{N}$-aminophthalimides and substituted aldehydes in ethanol. Moreover N-aminophthalimides $\quad(50-70 \%$ yield) were synthesized by reactions of corresponding phthalimides and hydrazine hydrate. Such compounds were characterized by different physico-chemical techniques like, melting point, elemental analysis, multinuclear NMR $(1 \mathrm{H}, 13 \mathrm{C})$.The synthesized compounds were screened for antibacterial and antifungal activities. The explorations of the biological properties of the compounds are mentioned in this paper.
\end{abstract}

Keywords N-aminophthalimide, Schiff Bases, Antimicrobial Activity, Aromatic Aldehydes, Azomethine

\section{Introduction}

Recent years have witnessed a great deal of interest in the synthesis and characterisation of Schiff bases [1]. They have a wide variety of applications in various fields, e.g., biological, inorganic and analytical chemistry [2-6]. They are used in optical and electrochemical sensors as well as in various chromatographic methods, to enable detection of enhance selectivity and sensitivity [7-9]. They possess excellent characteristics structural similarities with natural biological substances. Relatively easy preparation procedures and the synthetic flexibility enables design of suitable structural properties [10-16]. Schiff bases are also effective corrosion inhibitor because of ability of forming monolayer on the surface to be protected due to interaction with $\mathrm{C}=\mathrm{N}$ by electron transfer, chemisorptions [17-19]. In addition to this the atoms of the benzene rings create multiple adsorption sites for the inhibitor in forming stable monolayer formation. Imine linkage formed between the aldehyde derived from vitamin A and the protein opsin present in the retina of the eye plays a vital role in the chemistry of vision. Vitamin $\mathrm{B}_{6}$ serves as a coenzyme and is capable to form an imine with amino acid of protein. This is helpful in the transfer of the amino group from one amino acid to another, the transamination reactions, which is important in the metabolism and the biosynthesis of amino acids. Reports have shown that the presence of a lone pair of electrons in $\mathrm{sp}^{2}$ hybridized orbital of nitrogen atom present on the azomethine group has a vital role in exhibiting chemical and biological importance [20]. Schiff bases have been reported in their biological properties, such as, antibacterial, antifungal activities [21-24]. They are active against a wide range of organisms for example; Candida albicans, Escherichia coli, Staphylococcus aureus, Bacillus polymyxa, Trichophyton gypseum, Mycobacteria, Erysiphe graminis and Plasmopara viticola [25-28]. Ortho--phenylenediamine Schiff bases show clinical properties [29]. Isatin Schiff bases were reported to possess antiviral, anti-HIV, antiprotozoal and anthelmintic activities [30]. They also exhibit significant anticonvulsant activity, apart from other pharmacological properties [31]. Certain cobalt Schiff base complexes are potent antiviral agents [32]. Schiff bases derived from 4-dimethylamine benzaldehyde shows antibacterial activity, in medicines used as antibodies and anti-inflammatory agents [33-38]. All these facts prompted us to extend such works and we focused to synthesise Schiff bases with heterocyclic moieties such as phthalimides [39]. We also planned to screen such newly synthesized Schiff bases against microbial activities.

Following is the schematic diagram for preparation of Schiff bases which we followed: 
STEP (I)<smiles>[R2]c1ccc2c(c1[R])C(=O)NC2</smiles>

$1(a-d)$

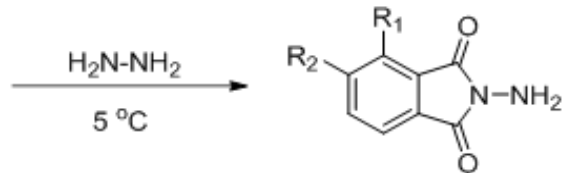

2(a-d)

$$
\begin{array}{ll}
\text { 1a. } R_{1}=\mathrm{NO}_{2}, & \mathrm{R}_{2}=\mathrm{H} \\
\text { 1b. } \mathrm{R}_{1}=\mathrm{Br}, & \mathrm{R}_{2}=\mathrm{H} \\
\text { 1c. } \mathrm{R}_{1}=\mathrm{H}, & \mathrm{R}_{2}=\mathrm{NO}_{2} \\
\text { 1d. } \mathrm{R}_{1}=\mathrm{H}, & \mathrm{R}_{2}=\mathrm{Br}
\end{array}
$$

$$
\begin{array}{ll}
\text { 2a. } \mathrm{R}_{1}=\mathrm{NO}_{2}, & \mathrm{R}_{2}=\mathrm{H} \\
\text { 2b. } \mathrm{R}_{1}=\mathrm{Br}, & \mathrm{R}_{2}=\mathrm{H} \\
\text { 2c. } \mathrm{R}_{1}=\mathrm{H}, & \mathrm{R}_{2}=\mathrm{NO}_{2} \\
\text { 2d. } \mathrm{R}_{1}=\mathrm{H}, & \mathrm{R}_{2}=\mathrm{Br}
\end{array}
$$

Figure 1. Synthesis of $\mathrm{N}$-aminophthalimides from phthalimides

STEP (II)<smiles>[R]c1ccc2c(c1Br)C(=O)N(N)C2=O</smiles>

2(a-d)<smiles>[Y4]c1c([Y4])c([Y4])c(C=O)c([Y4])c1[Y]</smiles>

3(a-d)

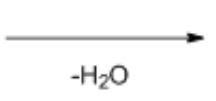<smiles>[R]c1ccc2c(c1[R])C(=O)N(/N=C/c1c([Y4])c([X])c([Y4])c([Y4])c1[Y4])C2=O</smiles>

$4(a-p)$

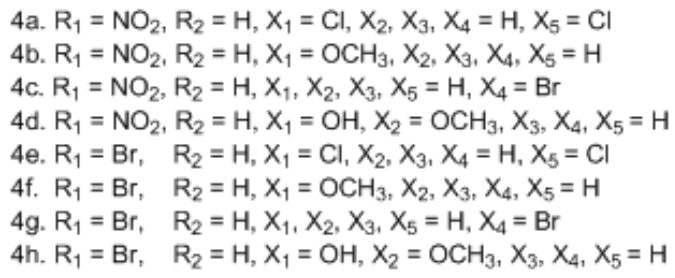

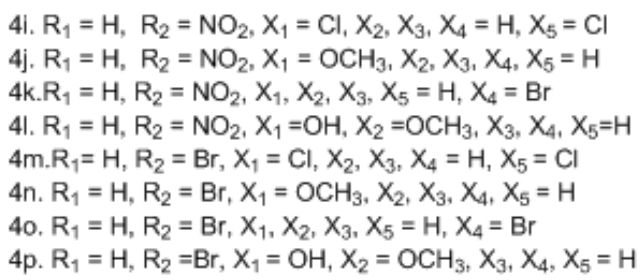

Figure 2. Synthesis of Schiff bases from $\mathrm{N}$-aminophthalimides

The formation of compounds was confirmed on the basis of elemental analysis, IR and PMR spectroscopical data.

\section{Experimental}

All the chemicals and reagents used were of AR quality. Materials used for the synthesis of the reported compounds were from reputed companies. Traditional method was used in the preparation of Schiff bases. Melting points were taken in open capillaries and are uncorrected. Purity of compounds was monitored on silica gel ' $G$ ' coated TLC plates. The IR spectra was taken on 157 spectrophotometer in $\mathrm{KBr}$ and PMR spectra on a varian A $60 \mathrm{D}$ instrument using TMS as internal standard.

\section{Preparation of 3- and 4-Substituted Phthalimides (1a-1d)}

3-and 4-nitrophthalimides were prepared by heating corresponding nitrophthalic acid with ammonium carbonate [39]. 4-aminophthalimide (m.p. $264^{\circ} \mathrm{C}$,d) was obtained as yellow crystals by stirring 4- nitrophthalimide (11.20 g, 0.11 mol) with a solution of stannous chloride $(84 \mathrm{~g}, 0.11 \mathrm{~mol})$ in $\mathrm{HCl}(450 \mathrm{ml})$ and water $(150 \mathrm{ml})$, cooling the resulting solid at $0^{\circ} \mathrm{C}$, washing it with hot water and crystallised as yellow needles. Similarly 3 -aminophthalimide ( m.p. $270^{\circ} \mathrm{C}$ ) was prepared in the same way which was yellow powder. These compounds were converted into 3- and 4-bromosubstituted derivatives by Sandmeyer reaction using cuprous bromide and $\operatorname{HBr}[40]$.

\section{Preparation of N-Aminophthalimides-(2a-2d)}

1:1 mole of phthalimides (1a-1d) and hydrazine in 90-100 $\mathrm{ml}$ of absolute ethanol were stirred under water-ice bath at $0-5{ }^{\circ} \mathrm{C}$ for $25 \mathrm{~min} .1 .5-2 \mathrm{ml}$ of $99 \%$ hydrazine were added to flask drop by drop. The mixture was well stirred at $0-5^{\circ} \mathrm{C}$ for $2.30 \mathrm{~h}$. Gradually viscosity of reaction medium increased with time. $60-80 \mathrm{ml}$ of ice-water was added to the reaction mixture. It was then filtered with vacuum pump. The precipitate obtained was washed with $5 \mathrm{ml}$ of water and dried well. The yield of the compounds was $50-73 \%$. 
Table 1. Physical Data of Compounds 4a-4p

\begin{tabular}{|c|c|c|c|c|c|c|c|c|}
\hline \multirow{2}{*}{ Entry } & \multirow{2}{*}{$\begin{array}{l}\text { Molecular } \\
\text { Formula }\end{array}$} & \multirow{2}{*}{ State } & \multirow{2}{*}{$\begin{array}{l}\text { Melting Point } \\
\left.\text { ( }{ }^{\circ} \mathrm{C}\right)\end{array}$} & \multirow{2}{*}{ Color } & \multirow{2}{*}{ Yield (\%) } & \multicolumn{3}{|c|}{$\%$ of Elements } \\
\hline & & & & & & $\mathrm{C}$ & $\mathrm{H}$ & $\mathrm{N}$ \\
\hline \multirow{2}{*}{$4 a$} & \multirow{2}{*}{$\mathrm{C}_{15} \mathrm{H}_{7} \mathrm{~N}_{3} \mathrm{O}_{4} \mathrm{Cl}_{2}$} & \multirow{2}{*}{ Solid } & \multirow{2}{*}{$177-179$} & \multirow{2}{*}{ Yellow } & \multirow{2}{*}{63} & 49.01 & 2.01 & 11.71 \\
\hline & & & & & & 49.45 & 1.92 & 11.54 \\
\hline \multirow{2}{*}{$4 b$} & \multirow{2}{*}{$\mathrm{C}_{16} \mathrm{H}_{11} \mathrm{~N}_{3} \mathrm{O}_{5}$} & \multirow{2}{*}{ Solid } & \multirow{2}{*}{$135-137$} & \multirow{2}{*}{ Yellow } & \multirow{2}{*}{71} & 59.28 & 3.47 & 13.01 \\
\hline & & & & & & 59.08 & 3.38 & 12.92 \\
\hline \multirow{2}{*}{$4 \mathrm{c}$} & \multirow{2}{*}{$\mathrm{C}_{15} \mathrm{H}_{8} \mathrm{~N}_{3} \mathrm{O}_{4} \mathrm{Br}$} & \multirow{2}{*}{ Solid } & \multirow{2}{*}{$127-129$} & \multirow{2}{*}{ White } & \multirow{2}{*}{73} & 48.31 & 2.18 & 11.41 \\
\hline & & & & & & 48.13 & 2.14 & 11.23 \\
\hline \multirow{2}{*}{$4 d$} & \multirow{2}{*}{$\mathrm{C}_{16} \mathrm{H}_{11} \mathrm{~N}_{3} \mathrm{O}_{6}$} & \multirow{2}{*}{ Solid } & & & & 56.25 & 3.13 & 12.12 \\
\hline & & & $231-232$ & Yellow & 62 & 56.31 & 4.23 & 12.32 \\
\hline & $\mathrm{C} \mathrm{C}_{2} \mathrm{~N}_{2} \mathrm{O} \mathrm{BrCl}_{2}$ & & & Pale & 65 & 45.42 & 1.82 & 7.08 \\
\hline 30 & $107=2=2$ & Solid & $100-105$ & yellow & & 45.23 & 1.76 & 7.06 \\
\hline$\Delta f$ & & & & Cream & & 53.81 & 3.09 & 8.1 \\
\hline & 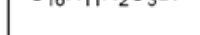 & Solia & & & 10 & 53.48 & 3.06 & 7.8 \\
\hline & & & & & 61 & 44.28 & 1.91 & 6.81 \\
\hline 49 & 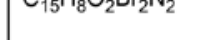 & Somats & $120-100$ & yellow & & 44.12 & 1.96 & 6.86 \\
\hline$A \mathrm{~h}$ & & Colid & & Pale & & 50.88 & 2.98 & 7.54 \\
\hline$m$ & $\mathrm{C}_{16} \mathrm{C}_{11} 1^{\mathrm{N}_{2}} \mathrm{U}_{4} \mathrm{DI}$ & Solic & $214-217$ & yellow & 50 & 51.20 & 2.93 & 7.47 \\
\hline & & & & & & 49.81 & 1.98 & 11.71 \\
\hline $4 i$ & $\mathrm{C}_{15} \mathrm{H}_{7} \mathrm{~N}_{3} \mathrm{O}_{4} \mathrm{Cl}_{2}$ & Solid & $182-184$ & White & 50 & 49.45 & 1.92 & 11.54 \\
\hline & & & & & & 59.28 & 3.27 & 12.85 \\
\hline $4 j$ & $\mathrm{C}_{16} \mathrm{H}_{11} \mathrm{~N}_{3} \mathrm{O}_{5}$ & Solid & $165-167$ & $\begin{array}{l}\text { Deep } \\
\text { yellow }\end{array}$ & 68 & 59.08 & 3.38 & 12.92 \\
\hline & & & & & & 48.25 & 2.08 & 11.28 \\
\hline $4 \mathrm{k}$ & $\mathrm{C}_{15} \mathrm{H}_{8} \mathrm{~N}_{3} \mathrm{O}_{4} \mathrm{Br}$ & Solid & $129-131$ & Cream & 62 & 48.13 & 2.14 & 11.23 \\
\hline 4! & & & & Yollow & 74 & 56.15 & 3.18 & 12.42 \\
\hline 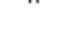 & $U_{16} \cap_{11} N_{3} U_{6}$ & Solild & $219-221$ & Tenow & 14 & 56.31 & 4.23 & 12.32 \\
\hline & & & & & & 45.12 & 1.88 & 7.10 \\
\hline $4 m$ & $\mathrm{U}_{15} \cap_{7} \mathrm{I}_{2} \mathrm{U}_{2} \mathrm{DICl}_{2}$ & Solla & $156-158$ & While & $6 r$ & 45.23 & 1.76 & 7.06 \\
\hline $4 n$ & $\mathrm{C}-\mathrm{H} \mathrm{NaO} \mathrm{Br}$ & & $145-147$ & Pale & 71 & 53.68 & 3.12 & 7.97 \\
\hline in & $C_{16} \cap_{11} \mathbb{N}_{2} \mathrm{O}_{3}$ & Solid & $140-14 t$ & yellow & 71 & 53.48 & 3.06 & 7.8 \\
\hline 40 & 0 & & $115-117$ & Pale & 52 & 44.19 & 1.85 & 6.91 \\
\hline 40 & $\mathrm{C}_{15} \mathrm{H}_{8} \mathrm{U}_{2} \mathrm{BI}_{2} \mathrm{~N}_{2}$ & Solid & (1) & yellow & 02 & 44.12 & 1.96 & 6.86 \\
\hline & & & & White & & 51.12 & 2.88 & 7.58 \\
\hline $4 p$ & $U_{16} \Pi_{11} 11_{2} V_{4} U_{4}$ & solia & $201-\angle 09$ & & $6 r$ & 51.20 & 2.93 & 7.47 \\
\hline
\end{tabular}

\section{Preparation of Schiff Bases (4a-4p)}

The compounds 2a-2d were condensed with aromatic aldehydes, 3a-3d, to produce the corresponding Schiff bases 4a-4p. Equimolar quantities of the $\mathrm{N}$-aminophthalimides $(0.01 \mathrm{~mol})$ and aromatic aldehydes $(0.01 \mathrm{~mol})$ were taken in 80-100 ml of xylene. Anhydrous $\mathrm{ZnCl}_{2}$ (3-5 g) was added to this solution. The mixture was refluxed for $6-8 \mathrm{~h}$. The crystals were separated and filtered under suction. The mother liquor of the above reactions were diluted with dry ether $(50-70 \mathrm{ml})$ and then saturated with dry $\mathrm{HCl}$ gas. Viscous liquid formed were separated and exposed to the atmosphere which turned into dark solid with metallic lustre. The compounds crystallized from alcohol have white, yellow or creamy colour crystal appearance in general. Melting points were determined, yield 50-78\%. 


\section{Result and Discussion}

\section{Melting Points}

It is expected that there are intramolecular hydrogen bonding in compounds $\mathbf{4 d ,} \mathbf{4 h}, \mathbf{4 l}$ and $\mathbf{4 p}$ hydroxy groups and so the melting point of these compounds (231-32, 214-15,219-21 and 207-9 ${ }^{\circ} \mathrm{C}$, respectively), are higher than those of the other Schiff bases.

\section{Spectral Analysis}

The IR spectra of the Schiff bases show medium or strong bands at $1615-1650 \mathrm{~cm}^{-1}$ assigned to $\mathrm{C}=\mathrm{N}$ stretching mode. The absence of bands characteristic of $(\mathrm{C}=\mathrm{O})$ and primary amine $\left(\mathrm{NH}_{2}\right)$ confirms the formation of the proposed Schiff base framework. The broad bands between 2800 and 2400 $\mathrm{cm}^{-1}$ in the spectra of the compounds $4 \mathrm{~d}, 4 \mathrm{~h}, \mathbf{4 l}$ and $\mathbf{4 p}$ have indicated the formation of the $\mathrm{OH} \cdots \mathrm{N}$ intramolecular hydrogen bond between the salicyl part $\mathrm{OH}$ proton and the nitrogen atoms. The $\mathrm{C}-\mathrm{O}$ stretching vibrations appear at the $1251-1298 \mathrm{~cm}^{-1}$ range as strong bands. The $\mathrm{C}-\mathrm{Cl}$ stretching vibration is seen at the range $610-648 \mathrm{~cm}^{-1}$ as medium or weak bands for all of the compounds. In the IR spectrum of 4a-4d and 4i-4l, the strong or medium bands at $1549-1528 \mathrm{~cm}^{-1}$ and at $1360-1365 \mathrm{~cm}^{-1}$ can be assigned to the symmetric and asymmetric vibrations of $\mathrm{NO}_{2}$, respectively. The higher $\boldsymbol{\delta}$ values (in NMR spectra using $\mathrm{CDCl}_{3}$ ) of the Schiff bases also confirm their formation and the presence of intra-molecular hydrogen bondings in the said compounds 4d,4h,4l and 4p (13.64,13.95,13.93 and 13.87 respectively). Methoxy group $\left(\mathrm{OCH}_{3}\right)$ in the compounds $\mathbf{4 b}, \mathbf{4 d}, \mathbf{4 f}, \mathbf{4 h}, \mathbf{4 j}, \mathbf{4 l}, \mathbf{4 n}$ and $\mathbf{4 p}$ appears as singlet ( $\boldsymbol{\delta}$ values 3.82-3.88) and is also supported by IR spectra $\left(v_{\max } 1170-1175 \mathrm{~cm}^{-1}\right)$.

Table 2. Spectral Data (I.R. and PMR) for $4 a-4 p$

\begin{tabular}{|c|c|c|}
\hline & IR Spectra $\left(\mathrm{KBr} \mathrm{cm}^{-1}\right)$ & PMR Spectra ( $\delta$ ) values \\
\hline \multirow{2}{*}{ 4a. $\mathrm{C}_{15} \mathrm{H}_{7} \mathrm{~N}_{3} \mathrm{O}_{4} \mathrm{Cl}_{2}$} & 1365, 1528(nitro),1260(C-O), & $6.4 \mathrm{~d}, 6.7 \mathrm{t}, 7.2 \mathrm{~d}, 7.8 \mathrm{~d}$ \\
\hline & 1792(OCN),1640(N=C), 648(C-Cl) & $8.96 \mathrm{~s}(\mathrm{~N}=\mathrm{CH})$ \\
\hline \multirow[t]{2}{*}{ 4b. $\mathrm{C}_{16} \mathrm{H}_{11} \mathrm{~N}_{3} \mathrm{O}_{5}$} & 1360, 1549(nitro),1258(C-O), & 6.3d,6.4t,6.7t,7.3d,7.5t \\
\hline & $1170\left(\mathrm{OCH}_{3}\right), 1797(\mathrm{OCN}), 1640(\mathrm{~N}=\mathrm{C})$ & $3.82 \mathrm{~s}\left(\mathrm{OCH}_{3}\right), 8.97 \mathrm{~s}(\mathrm{~N}=\mathrm{CH})$ \\
\hline \multirow[t]{3}{*}{ 4c. $\mathrm{C}_{15} \mathrm{H}_{8} \mathrm{~N}_{3} \mathrm{O}_{4} \mathrm{Br}$} & 1362,1546(nitro),1287(C-O), & $6.50 \mathrm{t}, 6.62 \mathrm{t}, 7.2 \mathrm{~d}, 7.5 \mathrm{t}, 8.0 \mathrm{~d}$ \\
\hline & $1792(\mathrm{OCN}), 1625(\mathrm{~N}=\mathrm{C})$ & $8.2 \mathrm{~d}(\mathrm{Ar}-\mathrm{H}), 8.96 \mathrm{~s}(\mathrm{~N}=\mathrm{CH})$ \\
\hline & $540(\mathrm{C}-\mathrm{Br})$ & \\
\hline \multirow[t]{3}{*}{ 4d. $\mathrm{C}_{16} \mathrm{H}_{11} \mathrm{~N}_{3} \mathrm{O}_{6}$} & 1366, 1532 (nitro),1295(C-O), & $6.4 \mathrm{t}, 6.7 \mathrm{t}, 7.21 \mathrm{~d}, 7.5 \mathrm{~s}, 7.8 \mathrm{t}$ \\
\hline & $\begin{array}{c}\text { 1792(OCN),1640(N=C), 630(C-Cl), } \\
2800 \text { (OH hydrogen bonding) }\end{array}$ & $8.96 \mathrm{~s}(\mathrm{~N}=\mathrm{CH})$ \\
\hline & $11.74\left(\mathrm{OCH}_{3}\right), 3280(\mathrm{OH})$ & $\begin{array}{c}\text { 9.92s }(\mathrm{OH}), 3.88 \mathrm{~s}\left(\mathrm{OCH}_{3}\right) \\
\text { 13.64(OH hydrogen bonding) }\end{array}$ \\
\hline \multirow[t]{2}{*}{ 4e. $\mathrm{C}_{15} \mathrm{H}_{7} \mathrm{~N}_{2} \mathrm{O}_{2} \mathrm{BrCl}$} & 1295 (C-O), 625,640 (C-Cl),578(Br) & $6.4 \mathrm{~d}, 6.7 \mathrm{~d}, 7.2 \mathrm{t}, 7.8 \mathrm{t}$ \\
\hline & $1780(\mathrm{OCN}), 1630(\mathrm{~N}=\mathrm{C})$ & $8.96 \mathrm{~s}(\mathrm{~N}=\mathrm{CH})$ \\
\hline \multirow[t]{2}{*}{ 4f. $\mathrm{C}_{16} \mathrm{H}_{11} \mathrm{~N}_{2} \mathrm{O}_{3} \mathrm{Br}$} & 1269 (C-O), 621,568 (C-Br) & 6.5d,6.7t,6.9t,7.3t,7.5d, \\
\hline & $1171\left(\mathrm{OCH}_{3}\right), 1778(\mathrm{OCN}), 1615(\mathrm{~N}=\mathrm{C})$ & $3.41 \mathrm{~s}\left(\mathrm{OCH}_{3}\right) 8.96 \mathrm{~s}(\mathrm{~N}=\mathrm{CH})$ \\
\hline \multirow[t]{2}{*}{ 4g. $\mathrm{C}_{15} \mathrm{H}_{8} \mathrm{O}_{2} \mathrm{Br}_{2} \mathrm{~N}_{2}$} & $1277(\mathrm{C}-\mathrm{O}), 1771(\mathrm{OCN})$ & 6.50t,6.7d,7.23d,7.4d,7.8t,8.1d \\
\hline & 1660(N=C), 587(C-Br)) & $8.96 \mathrm{~s}(\mathrm{~N}=\mathrm{CH})$ \\
\hline \multirow[t]{3}{*}{ 4h. $\mathrm{C}_{16} \mathrm{H}_{11} \mathrm{~N}_{2} \mathrm{O}_{4} \mathrm{Br}$} & 1260(C-O), 548(C-Br)) & $6.4 \mathrm{~s}, 6.7 \mathrm{t}, 7.4 \mathrm{~d}, 7.6 \mathrm{~d}, 7.8 \mathrm{t}$ \\
\hline & $1792(\mathrm{OCN}), 1640(\mathrm{~N}=\mathrm{C})$ & $8.96(\mathrm{~N}=\mathrm{CH})$ \\
\hline & $\begin{array}{c}3165(\mathrm{OH}), 1170\left(\mathrm{OCH}_{3}\right), \\
2598 \text { (OH hydrogen bonding) }\end{array}$ & $\begin{array}{c}\text { 9.93s }(\mathrm{OH}), 3.87 \mathrm{~s}\left(\mathrm{OCH}_{3}\right) \\
13.95 \mathrm{~s} \text { (OH hydrogen bonding) }\end{array}$ \\
\hline \multirow[t]{2}{*}{ 4i. $\mathrm{C}_{15} \mathrm{H}_{7} \mathrm{~N}_{3} \mathrm{O}_{4} \mathrm{Cl}_{2}$} & 1365, 1528(nitro),1260(C-O), & $6.4 \mathrm{~d}, 6.7 \mathrm{~d}, 7.5 \mathrm{~s}, 7.8 \mathrm{t}$ \\
\hline & $1792(\mathrm{OCN}), 1640(\mathrm{~N}=\mathrm{C}), 648(\mathrm{C}-\mathrm{Cl})$ & $8.1 \mathrm{~d}(\mathrm{Ar}-\mathrm{H}), 8.96 \mathrm{~s}(\mathrm{~N}=\mathrm{CH})$ \\
\hline \multirow[t]{2}{*}{ 4j. $\mathrm{C}_{16} \mathrm{H}_{11} \mathrm{~N}_{3} \mathrm{O}_{5}$} & 1365, 1528(nitro),1260(C-O), & 6.4d,6.7d,6.90d,7.10d,,7.8t \\
\hline & $1171\left(\mathrm{OCH}_{3}\right), 1792(\mathrm{OCN}), 1640(\mathrm{~N}=\mathrm{C}), 648(\mathrm{C}-\mathrm{Cl})$ & $3.85 \mathrm{~s}\left(\mathrm{OCH}_{3}\right), 8.96 \mathrm{~s}(\mathrm{~N}=\mathrm{CH})$, \\
\hline \multirow[t]{3}{*}{ 4k. $\mathrm{C}_{15} \mathrm{H}_{8} \mathrm{~N}_{3} \mathrm{O}_{4} \mathrm{Br}$} & 1362,1546(nitro),1287(C-O), & $6.50 \mathrm{t}, 6.72 \mathrm{~d}, 7.21 \mathrm{t}, 7.6 \mathrm{~d}, 7.82 \mathrm{~s}$ \\
\hline & 1792(OCN), & $8.96 \mathrm{~s}(\mathrm{~N}=\mathrm{CH})$ \\
\hline & $1625(\mathrm{~N}=\mathrm{C})$ & \\
\hline \multirow[t]{3}{*}{ 4l. $\mathrm{C}_{16} \mathrm{H}_{11} \mathrm{~N}_{3} \mathrm{O}_{6}$} & 1366, 1532 (nitro),1295(C-O), & 6.4d,6.7s,7.2d,7.4d,7.6d,7.9s \\
\hline & $1792(\mathrm{OCN}), 1640(\mathrm{~N}=\mathrm{C})$ & $8.96 \mathrm{~s}(\mathrm{~N}=\mathrm{CH})$ \\
\hline & $\begin{array}{c}1175\left(\mathrm{OCH}_{3}\right), 3280(\mathrm{OH}), \\
2800(\mathrm{OH} \text { hydrogen bonding) }\end{array}$ & $\begin{array}{c}\text { 9.92s }(\mathrm{OH}), 3.85 \mathrm{~s}\left(\mathrm{OCH}_{3}\right), \\
\text { 13.93s (OH hydrogen bonding) }\end{array}$ \\
\hline \multirow[t]{2}{*}{ 4m. $\mathrm{C}_{15} \mathrm{H}_{7} \mathrm{~N}_{2} \mathrm{O}_{2} \mathrm{BrC}$} & 1280 (C-O), 620,644 (C-Cl),585(C-Br) & $6.2 \mathrm{~s} .4 \mathrm{~d}, 6.7 \mathrm{~d}, 7.2 \mathrm{~d}, 7.51 \mathrm{~d}, 7.8 \mathrm{t}$ \\
\hline & $1786(\mathrm{OCN}), 1615(\mathrm{~N}=\mathrm{C})$ & $8.96(\mathrm{~N}=\mathrm{CH})$ \\
\hline \multirow[t]{2}{*}{ 4n. $\mathrm{C}_{16} \mathrm{H}_{11} \mathrm{~N}_{2} \mathrm{O}_{3} \mathrm{Br}$} & $1280(\mathrm{C}-\mathrm{O}), 544(\mathrm{C}-\mathrm{Br})$ & $6.4 \mathrm{~d}, 6.7 \mathrm{t}, 7.2 \mathrm{~s}, 7.8 \mathrm{t}$ \\
\hline & $1172\left(\mathrm{OCH}_{3}\right), 786(\mathrm{OCN}), 1615(\mathrm{~N}=\mathrm{C})$ & $3.78\left(\mathrm{OCH}_{3}\right), \quad 8.94 \mathrm{~s}(\mathrm{~N}=\mathrm{CH})$ \\
\hline \multirow[t]{2}{*}{ 4o. $\mathrm{C}_{15} \mathrm{H}_{8} \mathrm{O}_{2} \mathrm{Br}_{2} \mathrm{~N}_{2}$} & 1277(C-O), 1771(OCN), & 6.50t,6.70t,7.24d, 7.82s, \\
\hline & $1660(\mathrm{~N}=\mathrm{C}), 610,589(\mathrm{C}-\mathrm{Br}))$ & $8.96 \mathrm{~s}(\mathrm{~N}=\mathrm{CH})$ \\
\hline \multirow{3}{*}{ 4p. $\mathrm{C}_{16} \mathrm{H}_{11} \mathrm{~N}_{2} \mathrm{O}_{4} \mathrm{Br}$} & 1260(C-O), 548(C-Br) & 6.4s,6.7t,7.4d,7.6d,7.86d \\
\hline & $1792(\mathrm{OCN}), 1640(\mathrm{~N}=\mathrm{C})$ & 8.1d(Ar-H ,8.96( $\mathrm{N}=\mathrm{CH}), 9.92 \mathrm{~s}(\mathrm{OH}), 3.82\left(\mathrm{OCH}_{3}\right)$, \\
\hline & $\begin{array}{c}\text { 3089(OH),1171( }\left(\mathrm{OCH}_{3}\right), \\
2598 \text { (OH hydrogen bonding) }\end{array}$ & 13.87s (OH hydrogen bonding) \\
\hline
\end{tabular}


Table 3. Antibacterial Activity of Schiff's Bases ( $4 a-4 p)$

\begin{tabular}{|c|c|c|c|c|c|c|c|c|c|}
\hline \multirow{3}{*}{ Compounds } & \multicolumn{9}{|c|}{ Zone of Inhibition of Sample (in mm) } \\
\hline & \multicolumn{3}{|c|}{ E.coli } & \multicolumn{3}{|c|}{ B.subtilis } & \multicolumn{3}{|c|}{ S.aureus } \\
\hline & $20 \mathrm{mg} / \mathrm{ml}$ & $10 \mathrm{mg} / \mathrm{ml}$ & $5 \mathrm{mg} / \mathrm{ml}$ & $20 \mathrm{mg} / \mathrm{ml}$ & $10 \mathrm{mg} / \mathrm{ml}$ & $5 \mathrm{mg} / \mathrm{ml}$ & $20 \mathrm{mg} / \mathrm{ml}$ & $10 \mathrm{mg} / \mathrm{ml}$ & $5 \mathrm{mg} / \mathrm{ml}$ \\
\hline $4 a$ & 31 & 24 & 23 & 30 & 22 & 20 & 31 & 22 & 18 \\
\hline $4 \mathrm{~b}$ & 32 & 26 & 25 & 33 & 30 & 24 & 32 & 23 & 20 \\
\hline $4 c$ & 27 & 22 & 21 & 23 & 19 & 18 & 30 & 18 & 21 \\
\hline $4 d$ & 26 & 20 & 18 & 23 & 19 & 16 & 30 & 16 & 18 \\
\hline $4 \mathrm{e}$ & 22 & 19 & 18 & 17 & 11 & 10 & 28 & 18 & 16 \\
\hline $4 \mathrm{f}$ & 19 & 16 & 14 & 16 & 9 & 10 & 22 & 13 & 11 \\
\hline $4 \mathrm{~g}$ & 17 & 14 & 11 & 15 & 12 & 9 & 21 & 9 & 10 \\
\hline $4 \mathrm{~h}$ & 16 & 12 & 12 & 12 & 8 & 5 & 20 & 8 & 11 \\
\hline $4 \mathrm{i}$ & 31 & 24 & 23 & 26 & 22 & 20 & 31 & 18 & 16 \\
\hline $4 \mathrm{j}$ & 28 & 25 & 26 & 28 & 24 & 22 & 29 & 21 & 18 \\
\hline $4 \mathrm{k}$ & 24 & 20 & 22 & 22 & 18 & 15 & 28 & 16 & 16 \\
\hline $4 \mathrm{l}$ & 26 & 16 & 14 & 24 & 20 & 17 & 30 & 12 & 17 \\
\hline $4 \mathrm{~m}$ & 18 & 15 & 14 & 14 & 10 & 12 & 21 & 11 & 11 \\
\hline $4 n$ & 14 & 13 & 11 & 12 & 9 & 8 & 12 & 10 & 7 \\
\hline 40 & 13 & 12 & 11 & 10 & 8 & 5 & 18 & 8 & 8 \\
\hline $4 p$ & 11 & 10 & 8 & 9 & 7 & 4 & 15 & 8 & 6 \\
\hline
\end{tabular}

\section{Biological Activity}

\section{Antibacterial Activity Data of Compounds (in vitro)}

The bacterial cultures for B. subtilis, S. aureus, and E. coli were obtained from Department of Microbiology, College of Commerce, Patna. The bacterial cultures were incubated at $30 \pm 0.1^{\circ} \mathrm{C}$ for 24 hours by inoculation into nutrient agar. Schiff bases were stored dry at room temperature and dissolved in $20 \mathrm{mg} / \mathrm{ml}$ dimethylsulfoxide (DMSO). Antibacterial activities of each compound were evaluated by standard petridisc method [39, 41]. All plates were prepared with an equal thickness of nutrient agar. At the end period, inhibition zones formed on media were measured with a zone reader in millimetres. Similar results were also recorded for $10 \mathrm{mg} / \mathrm{ml}$ and $5 \mathrm{mg} / \mathrm{ml}$ respectively for each compound. A filter paper disc ( $5 \mathrm{~mm}$ diameter) was impregnated with the different Schiff's bases (at specific concentrations) and the disc was then placed on the nutrient agar in a petridish and left for $24 \mathrm{hrs}$ at $30 \pm 0.1^{\circ} \mathrm{C}$.

\section{Antifungal Activity of Compounds (in vitro), $4 a-4 p$}

Antifungal activities of compounds $\mathbf{4}$ were carried out at different concentrations using spores of Aspergillus flavus and Chalara corda by petridish method [42-44]. Spores of Aspergillus flavus suspended in $1.0 \mathrm{ml}$ of sterile water were mixed with $9.0 \mathrm{ml}$ of lukewarm molten agar and poured into a sterile petridish. After solidification of the agar, isolated single spores were marked under a microscope on an inverted petridish. Now, a circular agar area was cut off and aseptically transferred to PDA slants for growth at $25^{\circ} \mathrm{C}$. After one week of the growth, mycelia discs of $5 \mathrm{~mm}$ diameter were cut off from the maintained pure culture of the organism for inoculation into sterilized PDA medium in seven different petridishes for blotter disc studies. Small Whatman filter paper (no.44) discs of $15 \mathrm{~mm}$ were soaked in $5 \mathrm{mg} / \mathrm{ml}, 2.5 \mathrm{mg} / \mathrm{ml} \& 1.25 \mathrm{mg} / \mathrm{ml}$ concentrations of each Schiff's base in ethyl alcohol and sterile double distilled water were put in the middle of the inoculated petridishes one each separately. The petriplate containing the disc soaked in sterile distilled water served as the control. Paired petriplates were incubated at $25^{\circ} \mathrm{C}$ in inverted position for 6 days for the growth of the organism after which inhibitory effect of the compound was studied. Inhibitory zone was measured in each case.

\section{Conclusions}

All the newly formed Schiff'bases $\mathbf{4 a - 4 p}$ were found to be effective against bacterial and fungal activities to a greater extent. It was also found that the Schiff bases having nitro and chloro groups (electron withdrawing groups), $\mathbf{4 a - 4 d}$ and 4i-4l were more effective than the compounds having methoxy and hydroxyl groups (electron donating groups). The activity decreases with decrease in concentration.

\section{Acknowledgements}

The authors are thankful to the CDRI, Lucknow for the spectra IR, NMR, and CHN analyses and Department of Botany, College of Commerce, Arts and Science, Patna for biological activity tests. Financial support from the University Grants Commission (UGC), Kolkata, Minor project is gratefully acknowledged. 
Table 4. Antifungal Activity Data of Schiff's Bases [4a-4p]

\begin{tabular}{|c|c|c|c|c|c|c|}
\hline \multirow{3}{*}{ Compounds } & \multicolumn{6}{|c|}{ Zone of Inhibition of Sample in mm) } \\
\hline & \multicolumn{3}{|c|}{ Aspergillus flavus } & \multicolumn{3}{|c|}{ Chalara corda } \\
\hline & $5 \mathrm{mg} / \mathrm{ml}$ & $2.5 \mathrm{mg} / \mathrm{ml}$ & $1.25 \mathrm{mg} / \mathrm{ml}$ & $5 \mathrm{mg} / \mathrm{ml}$ & $2.5 \mathrm{mg} / \mathrm{ml}$ & $1.25 \mathrm{mg} / \mathrm{ml}$ \\
\hline $4 a$ & 42 & 25 & 18 & 38 & 28 & 12 \\
\hline $4 \mathrm{~b}$ & 47 & 29 & 22 & 36 & 26 & 16 \\
\hline $4 c$ & 40 & 21 & 14 & 30 & 21 & 14 \\
\hline $4 \mathrm{~d}$ & 39 & 20 & 16 & 32 & 22 & 12 \\
\hline $4 \mathrm{e}$ & 24 & 12 & 10 & 36 & 26 & 16 \\
\hline $4 \mathrm{f}$ & 26 & 10 & 8 & 34 & 24 & 14 \\
\hline $4 \mathrm{~g}$ & 22 & 14 & 12 & 36 & 23 & 13 \\
\hline $4 \mathrm{~h}$ & 20 & 12 & 21 & 22 & 21 & 11 \\
\hline $4 \mathrm{i}$ & 43 & 25 & 21 & 37 & 25 & 17 \\
\hline $4 \mathrm{j}$ & 47 & 28 & 21 & 38 & 24 & 16 \\
\hline $4 \mathrm{k}$ & 40 & 20 & 18 & 35 & 26 & 16 \\
\hline $4 l$ & 38 & 18 & 12 & 26 & 12 & 10 \\
\hline $4 \mathrm{~m}$ & 26 & 17 & 14 & 30 & 18 & 12 \\
\hline $4 n$ & 20 & 12 & 9 & 21 & 11 & 9 \\
\hline 40 & 18 & 11 & 8 & 20 & 8 & 7 \\
\hline $4 p$ & 17 & 8 & 6 & 21 & 7 & 5 \\
\hline
\end{tabular}

[11] Bhendkar, A.K., Vijay, K. \& Raul, A.W. [2004]. Acta Cien. Indica, 30C, 29.

\section{REFERENCES}

[1] Schiff, H.S. [1864], Ann. Chem. (Paris). 131,118.

[2] Cimerman, Z., Miljanic, S. \& Galic, N. [2000]. Croatica Chemica Acta, 73 (1), 81- 95.

[3] Singh, P., Goel, R. L. \& Singh, B. P. [1975]. J. Indian Chem. Soc., 52, 958.

[4] Perry, B. F., Beezer, A. E., Miles, R. J., Smith, B. W., Miller , J. \& Nascimento, M. G.[ 1988]. Microbois., 45, 181.

[5] Elmali, A., Kabak, M. \& Elerman, Y. [2000]. J.Mol. Struct., 477, 151.

[6] (a) Patel, P. R., Thaker, B. T. \& Zele, S.[1999]. Indian J. Chem., 38 A, 563. (b) Hodnett, E.M., Dunn, W. J., [1970], J.Med. Chem., 13, 768.

[7] Valcarcel, M. \& Laque de Castro, M. D.[1994]. "Flow-Through Biochemical Sensors", Elsevier, Amsterdam.

[8] Spichiger-Keller, U. [1998]. "Chemical Sensors and Biosensors for Medical and Biological Applications", WileyVCH, Weinheim.

[9] (a) Lawrence, J. F. \& Frei, R. W. [1976]. "Chemical Derivatization in Chromatography", Elsevier, Amsterdam. (b) Olie, G. H. and Olive, S. [194] Springer, Berlin.

[10] More, S.V., Dongarkhadekar, D.V., Chavan, R.N., Jadhav, W.N., Bhusare, S.R. and Pawar, R.P.[2002]. J. Indian Chem. Soc., 79, 768.
[12] Vaghasiya, Y.K., Nair, R.S., Baluja, M. \& Chanda, S.S. [2004]. J. Serb. Chem. Soc., 69, 991.

[13] Vashi, K. \& Naik, H.B. [2004]. Eur. J. Chem., 1, 272.

[14] Rhodes, J. H. Chen, Hall, S.R., Beesley, J.E., Jenkins, Collins D.C. P. \& Zheng, B. [1995]. Nature, 377, 71.

[15] Safwat, H.M., Ragab, F.A., Eid, N.M. \& Abdel, G.M. [1988]. Egypt.J. Pharm. Sci., 29, 99

[16] Mtrei, Yadawe, R. M. and Patil, S. ([1996]. Orient. J. Chem., 12, 101.

[17] Quan, Z., Chen, S. and Li, Y. [2001]. Corros. Sci., 43, 1071.

[18] Ashassi, Sorkhabi, Shabani, H. B., Aligholipour, B. and Seifzadeh, D. [2006] Appl. Surf. Sci., 252, 4039.

[19] Li, S., Chen, S., Ma, H., Yu, R. and Liu, D., [1999]. Corros. Sci, 41, 1273.

[20] (a) Aurea, E.,Maria da, G. N., Vanilde, G., Joseph Mand Astréa ,G. J. [1999] Braz. Chem. Soc., 10 ,60 (b) Kuzmin, V. E., Lozitsky, V. P., Kamalov, G. L., Lozitskaya ,R. N., Zheltvay, A. .I, Fedtchouk, A .S. and Kryzhanovsky, D. N. [2000]. Acta Biochemical Polonica., 47, 867.

[21] (a) Williams, D.R. [1972]. Chem. Rev., 72, 203. (b) Campos, A., Anacona, J.R. and Campos-Vallette, M.M. [1999]. Mian group Metal chem., 22, 283. (c) Arslan, N. S., Logoglu, E. and Sakiyan, I. [2003]. G.U.J. Sci., 16, 283.

[22] (a) Safwat, H.M., Ragab, F. A., Eid, N.M. \& Abdel, G.M. [1988]. Egypt. J. Pharm. Sci., 29, 99 (1988). (b) Bilgramy, K. S., Prasad, T., Sinha, K. K. \& Singh, P. [1980]. J. Indian Bot. 
Soc. 59: $123-12$.

[23] Davis, W., Roberts, J. J \& Ross, W. C. J. [1995]. J. Chem. Soc. 890.

[24] Dhar, M. L., Dhar, M. M., Dhawan, B. N., Mehrotra, B. N. \& Ray, C. [1968]. Indian J. Exp Biol.6: 232-248.

[25] Ebbole, D. J. [1996]. J. Genet.75, 361-374. and Barreiro, E.J.[ 2002]. Bioorg. Med. Chem. Lett., 12, pp. 1533.

[26] Siliva Regina Tozato Prado et al. [2004]. Z. Naturforsch, 59, pp.663.

[27] Sami, S. M, Dorr, R.T., Alberts, D.S., Solyom, A. M. and Remers, W.A. [2000] .J. Med. Chem., 43, 3067.

[28] Wang, T., Zhang, Y.H., Ji, H., Chen, Y.P. and Peng, S.X. [2008]. Chinese Chem. Lett., 19, 26.

[29] Sandmeyer, T. [1884].Berichte der deutschen chemischen Gesellschaft. 17(4), 2650-2653.

[30] Mahindra A.M. and Fisher, J.M. [1983].Nature (London), 303, 64.

[31] Pandeya, S.N., Yogecswari P., Sriram, D. [1999] Chemotherapy, 45, 192.

[32] Sawodny, W.J. and Riederer, M.[1977]. Angew. Chem. Int. Edn. Engi. 16, 859.

[33] Bottcher, A., Takeuchi, Simon, T. M.I., Meade T.J. and Gray, H.B., [1995].J. Inorg. Bio-Chem., 59, 221.

[34] Britovsek, G.L.P., Gibson, V.V., Mastroianni, S., Oakes, D.C.H., Redshaw, C., Solan, G.A. White, A.J.P., Williams, .J. [2001]. Eur. J. Inorg. Chem., 431, 2.

[35] Sun, B., Chen, J., Hu J.Y. [201], Lix., J. Chin. Chem. Soc, 12, 1043.
[36] Boghaei D.M. and Mohebi, S., [2002]. Tetrahederon, 58, 5357.

[37] Liu, S.Y., Nocera, D.G., [2006]. Tetrahedron Lett., 47, 1923.

[38] Budakoti A., Abid M. and Azam, A., [2006]. Eur. J.Med. Chem., 41, 63.

[39] (a) Sinha, G.K., Pandey, B., Singh, N.N.[1999]. J.Indian Chem. Soc., 76, pp. 110-11., (b) Sinha, Girish Kumar, Singh N.N. 2ooo, ChemInform ,Vol.31, Issue 18, page no May 2, Wiley Online Library, 111 River Street, Hoboken, NJ 07030-5774, North America (USA)); (c) Sinha, G.K., Narayan, S., Yadav, S.N., Priya, A.[2014].,'Synthesis and Biological Activity of Some Schiff's Bases(N-Aminophthalimide Series)', Advances in Scientific and Technological Research(ASTR), USA, [online] 1 (4), 149-160. (d) Sinha, Girish Kumar, Singh N.N.[1999], "Synthesis Of Some Nitrogen Mustards”, Journal Of Indian Chemical Society Calcutta,, Vol.76,February1999, pp.110-111.

[40] (a) Sandmeyer Reaction.” Chem. Rev. 40(2); 251-277.; (b) Doyle, M.P., Siegfried, B. and Dellaria, J.F. (1977), J. Org. Chem. 42 (14); 2426-2431.

[41] Atan M.S., Dzulkefly K.A., Aspollah S. Mohd., Anuar K. \& Vijay S. [2011]. "Isolation and antibacterial activity of alkaloids from Phaeanthusopthalmicus." Asian Journal of Chemistry. 23, 3824-3826.

[42] Verma R. S. and Imam S. A. [1973]. Indian J. Microbiol.13, pp. 45.

[43] Siliva Regina Tozato Prado et al. [2004]. Z. Naturforsch, 59, pp.663.

[44] Sami, S. M, Dorr, R.T., Alberts, D.S., Solyom, A. M. and Remers, W.A. [2000] .J. Med. Chem., 43,. 3067. 